\title{
Fentanyl transmucosal tablets: current status in the management of cancer-related breakthrough pain
}

This article was published in the following Dove Press journal:

Patient Preference and Adherence

22 June 2012

Number of times this article has been viewed

\author{
Eric Prommer \\ Brandy Ficek \\ Division of Hematology/Oncology, \\ Mayo Clinic College of Medicine, \\ Mayo Clinic Hospital, Scottsdale, \\ AZ, USA
}

\begin{abstract}
Breakthrough pain is a newly recognized pain category that was first described by Portenoy and Hagen in 1990. The term describes pain that increases in intensity to "break through" chronic pain that is being controlled by a scheduled opioid regimen. The development of fluctuations in pain intensity is challenging due to their unpredictable nature, rapid onset, and need for rapid treatment intervention. Breakthrough pain has been treated by using an extra opioid dose in addition to the scheduled opioid being used for pain. Recommendations for dose and frequency are based on expert opinion only, and have included dosing based on a percentage of the total opioid dose. Other recommendations include increasing the regularly scheduled opioid dose. Clinical trials have now focused on delivery of opioids that have both potency and a rapid onset of action. Lipophilic opioids have received a substantial amount of study due to their quick absorption and rapid onset of analgesia. Lipophilic opioids that have been studied to date include transmucosal fentanyl, sublingual fentanyl, intranasal sufentanil, and oral and sublingual methadone. Initial clinical trials have established the superiority of transmucosal fentanyl as a breakthrough analgesic when compared with immediate-release oral opioid formulations. Problems with bioavailability have led to a search for newer formulations of transmucosal delivery. Newer formulations, such as fentanyl transmucosal tablets, have been developed to ensure superior delivery for the patient suffering from breakthrough pain. The purpose of this paper is to discuss the current status of transmucosal tablet formulations for cancer breakthrough pain.
\end{abstract}

Keywords: fentanyl, transmucosal, tablets, pain, breakthrough, cancer

\section{Introduction}

Breakthrough pain (BTP) has been defined as "a transitory increase in pain intensity on a baseline pain of moderate intensity in patients receiving regularly administered analgesic treatment". ${ }^{1}$ BTP arises in $40 \%-80 \%$ of patients with chronic cancer pain. ${ }^{2}$ This pain has a rapid onset, of severe intensity, and is not effectively treated with shortacting opioids due to the slow onset of action of most orally administered immediaterelease formulations. ${ }^{3}$ BTP can occur frequently. Surveys of BTP show that the median number of episodes per day is four, with a time onset to maximum pain of 3 minutes and a 30-minute episode duration. ${ }^{1}$ BTP has a marked impact on quality of life and factors into the increased cost of medical care. ${ }^{2}$ Patients suffering from repeated BTP episodes have poor quality of life, including impaired sleep, impaired performance of their activities of daily living, and impaired overall well-being. BTP can worsen anxiety and depression, affect compliance with opioid therapy, and lead to poor medical outcomes. ${ }^{2}$ Patients with cancer-related BTP or uncontrolled pain experience more 
pain-related hospitalizations and emergency department visits and have increased treatment costs compared with patients without BTP. ${ }^{2}$ One report found that annual costs of cancer pain-related hospitalizations, emergency visits, and physician office visits was five times greater for patients with BTP. ${ }^{2}$

\section{Classification of breakthrough pain}

Table 1 lists the current classifications of cancer-related BTP. The most common subtypes are incident, idiopathic, spontaneous, and end-of-dose subtypes. ${ }^{4}$ Incident BTP is best characterized as pain provoked by movement, and accounts for nearly $50 \%$ of total BTP. This pain is provoked by musculoskeletal movements, such as coughing or turning over in bed. ${ }^{1}$ It is also important to remember that incident pain can arise from visceral smooth muscle, such as with bowel or bladder spasms, and can be especially unpredictable. The idiopathic subtype of BTP does not have an identifiable cause and lasts longer than incident pain. ${ }^{5}$ The end-of-dose subtype describes a clinical presentation where patients experience worsening pain before their scheduled dose of opioid. The cause and anatomical site of BTP is usually, but not always, the same as the baseline pain the patient experiences. ${ }^{6}$

\section{Pathophysiology of breakthrough pain arising in bone}

BTP arising from bone is now being viewed as mechanical allodynia. ${ }^{7}$ Bone is densely innervated by unmyelinated C-fibers and myelinated A- $\delta$ fibers, the endings of which are damaged by the direct effects of tumors, immune reactive cells, and their chemical mediators, as well as by acidosis produced in the tumor microenvironment. ${ }^{8}$ Tumor-derived products play a role in activating nerve impulses. These products include prostaglandins, tumor necrosis factor $\alpha$, endothelins, interleukins 1 and 6, epidermal growth factor, transforming growth factor- $\beta$, and platelet-derived growth factor. ${ }^{8}$ The effect of these tumor products is to enhance the conduction of nerve fibers, thus conducting nociceptive impulses centrally and to the spinal cord.

\section{Approaches to breakthrough pain}

BTP is managed by the use of a short-acting opioid in addition to the regularly scheduled opioid. Expert opinion recommends that the dose of breakthrough analgesic be based on a

Table I Breakthrough pain classification

Incident (predictable and unpredictable subtypes)

Idiopathic

End of dose percentage of the total (long-acting) opioid dose (10\%-20\%) and providing that dose every 1-2 hours as needed. Other recommendations include an increase in the breakthrough dose as the scheduled opioid dose is increased. There have been no studies evaluating the optimal breakthrough percentage dose. More consensus has been reached on the management of the end-of-dose subtype of BTP, which is treated by opioid dose escalation of the scheduled opioid. ${ }^{7}$ Matching the rapidity of the onset of BTP continues to be a substantial problem. Use of immediate-release morphine, with an onset of action of 20-30 minutes, ${ }^{9}$ cannot be quick enough to provide analgesia for the rapid forms of BTP. Parenteral therapy could provide rapid relief from this pain, ${ }^{10}$ but this route is not particularly convenient for the patient. Clinical trials have now focused on delivery of opioids that have both potency and a rapid onset of action. Lipophilic opioids have received a considerable amount of study due to their quick absorption and rapid onset of analgesia. Lipophilic opioids have the added advantage of being readily absorbed across multiple routes of administration. Lipophilic opioids that have been studied to date include transmucosal fentanyl, sublingual fentanyl, intranasal sufentanil, and oral and sublingual methadone. ${ }^{11-15}$ Fentanyl, which is a potent synthetic opioid, is used in the treatment of BTP. ${ }^{16}$ It has a high affinity for the $\mu$ opioid receptor, where, like morphine, it acts as a pure agonist. The association with the $\mu$ opioid receptor is rapid $\left(\mathrm{t}_{1 / 2}=2.5\right.$ minutes). Fentanyl interacts with the $\mu$ opioid receptor with a $\mathrm{Ki}$ of $2.9 \pm 0.2$, which indicates that this interaction is more potent than for morphine. Fentanyl has lower affinity for the $\delta$ and $\kappa$ opioid receptor, with Ki values of $180 \pm 18$ and $290 \pm 24$, respectively. ${ }^{17}$ Fentanyl is highly lipophilic and is easily absorbed by permeation through the mucosal surfaces. ${ }^{18}$ One available fentanyl formulation is in the form of fentanyl embedded in a matrix, which is placed on a stick that is held on the mucosa, allowing the fentanyl to be dissolved and absorbed across the mucosa. ${ }^{19}$ Unfortunately, this method of delivery is associated with a large proportion of the active fentanyl being swallowed. ${ }^{20}$ This lowers the bioavailability of the transmucosal fentanyl formulation to approximately $47 \%$ compared with the intravenous route. ${ }^{20}$ Swallowing allows metabolism to occur in the intestine and liver by cytochrome P4503 A4. ${ }^{21}$ Despite these drawbacks, the transmucosal fentanyl formulation can deliver rapid analgesia within 15 minutes. $^{22}$ The half-life is about 7 hours. ${ }^{23}$ Sublingual fentanyl is another route of delivery for incident pain. When delivered as a sublingual solution, there is a rapid onset of analgesic action, but swallowing, which leads to decreased bioavailability, is also a problem. ${ }^{24}$ 
The development of the transmucosal tablet has been shown to be an advance in the delivery of fentanyl. This dosage form was designed to encourage retention of the active substance with the mucosa, so as to increase contact time at the absorption site and avoid the potential intraindividual and interindividual variability resulting from swallowing. ${ }^{25}$ Onset of action is rapid (8-11 minutes after administration), with available preparations showing dose proportionality. ${ }^{26}$ The tablet formulations have been shown to be easy for patients to use. ${ }^{27}$

\section{Transmucosal tablet formulations}

For a tablet to be successful for absorption across the mucosa, among the most important characteristics are a short disintegration and dissolution time and prolonged contact time with the mucosa. ${ }^{27}$ Other important characteristics are small particle size and high solubility. ${ }^{28}$ If drug dissolution is incomplete or contact time with the mucosa is too short, there is a greater likelihood that the drug being swallowed will not be absorbed, decreasing bioavaiability. Thus, the ideal drug should be one that is rapidly dissolved and able to maintain a long contact time with the mucosa. ${ }^{27}$ To increase contact time, the concept of ordered mixtures was introduced. This is the mixing of a drug particle with a carrier unit, allowing both improved contact time and rapid dissolution and negating the effects of swallowing. ${ }^{29}$ The adherence of a drug particle to a carrier particle also facilitates dissolution. ${ }^{30}$ Improved adhesion can be accomplished with the addition of bioadhesives to the mixture. ${ }^{27}$ Provided the drug is instantly dissolved and able to permeate the mucous membranes easily, it will be rapidly absorbed at the administration site before there is a chance of it being swallowed..$^{25}$

\section{Pharmacology of fentanyl transmucosal tablets}

In healthy populations, six trials have evaluated the pharmacokinetics of the sublingual tablet. Studies have consisted of single doses, ${ }^{31}$ repetitive dosing, ${ }^{32}$ and bioequivalency trials, ${ }^{33}$ as well as bioavailability comparisons of the sublingual tablet with oral transmucosal fentanyl citrate at various doses..$^{34,35}$ The area under the curve and time taken to reach peak concentration $\left(\mathrm{T}_{\max }\right)$ are proportional to dose for single-dose tablets of $100-800 \mu \mathrm{g} .{ }^{31} \mathrm{The} \mathrm{T}_{\max }$ is approximately 35-45 minutes. ${ }^{31}$ Once above $800 \mu \mathrm{g}$, there is a loss of dose proportionality. ${ }^{31}$ There is a higher maximum plasma concentration $\left(\mathrm{C}_{\max }\right)$ when four tablets are given, as opposed to one tablet. ${ }^{33}$ It takes approximately 5 days to reach steady state when $400 \mu \mathrm{g}$ tablets are given on a 6-hourly schedule. ${ }^{36}$
When compared with oral transmucosal fentanyl citrate, it appears that the tablets enter the circulation at a faster rate and with higher concentration $\left(\mathrm{T}_{\max }, \mathrm{C}_{\max }\right.$, and area under the curve). ${ }^{35}$ When compared with oral transmucosal fentanyl citrate, the median $\mathrm{T}_{\max }$ was reached at 47 versus 91 minutes, respectively. ${ }^{35}$ Bioavailability was higher with the tablet as opposed to oral transmucosal fentanyl citrate, with the tablets having a bioavailability of $65 \%$ versus a bioavailability of $47 \% .^{33}$

\section{Pharmacology in mucositis}

Darwish et al examined the effect of mucositis on the absorption of the tablet formulation in an open-label Phase I study. ${ }^{37}$ Adult cancer patients currently on an opioid regimen equivalent to 60-1000 $\mathrm{mg}$ of oral morphine daily for at least one week were recruited for this study. Patients with oral mucositis were eligible if their mucositis was grade 1 to 3 on clinical examination or grade 1 to 2 on functional/symptomatic examination, according to the Common Terminology Criteria for Adverse Events grading system.

Patients were given one $200 \mu \mathrm{g}$ dose of buccal fentanyl tablets in the morning. The medication was self-administered and allowed to dissolve in the buccal mucosa without any manipulation for 10 minutes. If the tablet was not fully dissolved after this amount of time, patients then applied gentle massage to the cheek at the location of the tablet, followed by an additional 30 minutes to allow the tablet to dissolve fully. This process was similar for patients with oral mucositis, except that the least affected buccal area was chosen for location of placement of the buccal fentanyl tablets. In addition, patients with mucositis agreed to refrain from topical mucositis treatments one hour prior to and 8 hours after use of the study medication. Venous blood samples from all patients were collected prior to administration of the buccal fentanyl tablets and at time intervals of 10, 20, 30, 40, 45, and 50 minutes and 1, 2, 3, 4, 6, and 8 hours after placement of the buccal tablets. These samples were analyzed for fentanyl concentrations using high-performance liquid chromatography with tandem mass spectrometric detection.

Nineteen patients were enrolled in the study and 16 patients, including eight patients with and eight patients without mucositis, completed the data collection. In the patients with mucositis, the clinical grade was 1 for all eight patients, and the functional grade was 1 for all except one patient with a functional grade of 2 . In 14 of the 16 patients, the buccal fentanyl tablet was fully dissolved within the initial 30 minutes. When comparing the $\mathrm{C}_{\max }, \mathrm{T}_{\max }$, and area under the plasma concentration-time curve, there 
was no marked difference between patients with and without mucositis in absorption of the buccal fentanyl tablets.

Adverse events were recorded during the study. Three patients withdrew prior to receiving the study medication, including one secondary to a serious adverse event of intestinal obstruction. Of the 16 patients who received the buccal fentanyl tablet, four reported adverse effects including dizziness, nausea, anemia, and back pain. All reported that adverse effects were mild or moderate in severity. No adverse application site effects occurred and no changes in the mucosal examination were observed.

\section{Transmucosal fentanyl tablets: clinical trials}

Most of the clinical trials involving the transmucosal fentanyl tablet formulations have been placebo-controlled. The populations participating in these studies have been relatively healthy and without renal/hepatic impairment or other comorbidities. Criteria for responses vary between studies, so do not allow direct comparisons, and the approaches taken in the studies do not uniformly reflect clinical practice. The studies conducted so far have not involved special populations such as the elderly. There have been no comparisons of transmucosal fentanyl tablets with other transmucosal formulations. In addition, the studies have been sponsored by the manufacturer and so have potential for bias. ${ }^{38}$

\section{Clinical efficacy and safety: sublingual route \\ Phase II trial}

Lennernas and et al performed a proof-of-concept study to evaluate the efficacy and tolerability of sublingual fentanyl tablets for cancer BTP. ${ }^{39}$ All patients were adults with locally advanced or metastatic cancer who were regularly experiencing BTP. Patients were required to be opioid-tolerant and receiving a fixed-dose schedule of opioids equivalent to $30-1000 \mathrm{mg} /$ day oral morphine or $25-300 \mu \mathrm{g} / \mathrm{hour}$ transdermal fentanyl.

In this study, 38 patients were randomized to receive single doses of 100,200 , or $400 \mu \mathrm{g}$ of sublingual fentanyl or placebo, in random order, during four distinct pain episodes. These episodes were separated by a minimum of one day to avoid any carryover effects of the medication. Efficacy was measured using the pain intensity difference immediately prior to and after treatment at intervals of 5, 10, 15, 20, and 30 minutes. Pain intensity was evaluated by a $100 \mathrm{~mm}$ visual analog scale $(0=$ no pain, $100=$ worst conceivable pain $)$, where a clinically important decrease in pain intensity was defined as a decrease of more than $20 \mathrm{~mm}$. Secondary outcomes included a global assessment of treatment 60 minutes after dosing using a four-point scale ("none," "mild," "moderate," or "excellent") and need for rescue medication, which could be taken 30 minutes after study drug intake if pain relief was inadequate.

In total, 27 patients received at least one dose of sublingual fentanyl and 23 patients received all four doses. The results showed that a single $400 \mu \mathrm{g}$ dose of sublingual fentanyl was significantly more effective in reducing pain intensity than placebo $(P<0.0001)$ in the 23 patients who received all doses. An intention-to-treat analysis of all patients who received at least one dose also demonstrated significant variation in pain intensity differences between the $400 \mu \mathrm{g}$ sublingual fentanyl tablet and placebo $(P=0.007)$. This result was supported by secondary outcomes, including less requirement for rescue analgesia with sublingual fentanyl tablets than with placebo $(P=0.001)$. More patients rated their treatment as excellent with use of the $400 \mu \mathrm{g}$ fentanyl tablet than with placebo $(P=0.0146$.) The timing to significant relief was first evident at 15 minutes following administration $(P=0.005)$ and remained significant at 20 $(P=0.005)$ and 25 minutes $(P=0.02)$ post-administration. There was no significant difference between placebo and the $100 \mu \mathrm{g}$ and $200 \mu \mathrm{g}$ doses of sublingual fentanyl.

All 38 patients were included in the evaluation of tolerability and safety. A total of 15 adverse events were reported by 13 patients. The most common were pain and vomiting. Two events, nausea/vomiting and dizziness, were thought to be related to administration of sublingual fentanyl. There were no marked differences in adverse events between dosages of sublingual fentanyl. Three serious adverse events, progressive cancer, septicemia, and intense lower abdominal pain, were considered to be unrelated to sublingual fentanyl administration.

\section{Phase IV trial}

Uberall et al assessed the efficacy, impact on quality of life, and safety of sublingual fentanyl tablets in patients seen in routine clinical practice. ${ }^{40}$ Adult patients who had previously been receiving a fixed-schedule oral opioid regimen for cancer-related pain and who had been prescribed sublingual fentanyl disintegrating tablets for the first time were eligible for enrollment in this study.

This open-label Phase IV study including five study visits to assess baseline and treatment period data. The efficacy of fentanyl was evaluated using questionnaires to document 
BTP intensity on an 11-point scale ( $0=$ no pain, 10 strongest pain conceivable), time to first effect following use of fentanyl, time to maximum effect, comparison with previous analgesic therapy using a seven-point scale in five domains (speed of action, strength of action, duration of action, tolerability, and ease of handling), physical functioning with a modified version of the pain disability index, and emotional function using the Hospital Anxiety and Depression Scale. Tolerability was monitored with self-reported adverse events as well as by clinician observation.

A total of 217 patients were enrolled in this study, and 181 completed the entire 28-day observation period. The mean maximum intensity of BTP was significantly improved, from 7.8 at enrollment to 2.6 for episodes treated with sublingual fentanyl $(P<0.0001)$. The time to first effect was reported as less than 10 minutes in $82.8 \%$ of episodes. The time to maximum effect was less than 30 minutes in $63.2 \%$ of episodes. In comparison with previous medication regimens, $87.7 \%$ of patients reported improved speed of action with use of sublingual fentanyl. A majority of patients also favored sublingual fentanyl over their previous medication in terms of strength of action $(85.7 \%)$, duration of action (83.9\%), tolerability (88.6\%), and ease of handling (87.3\%). Quality of life indicators also improved over the course of this study. The mean combined modified version of the Pain Disability Index scores showed significant improvement in daily functioning at the end of the study visit with use of sublingual fentanyl $(P<0.0001)$. The percentage of patients experiencing painrelated disability decreased from time of enrollment to the end of the study ( $73 \%$ versus $12.1 \%, P<0.001)$. Scores on the Hospital Anxiety and Depression Scale showed a significant decrease in anxiety and depression during the study period. Abnormal levels of anxiety were present in 54.5\% patients at baseline, compared with $1.6 \%$ of patients at the end of the study $(P<0.0001)$. At baseline, $70.3 \%$ of patients were experiencing high levels of depression, compared with $15.6 \%$ at the end of the study $(P<0.0001)$.

Of the 217 patients enrolled, 33 (15.2\%) experienced at least one adverse event. Adverse events were considered likely to be related to the sublingual disintegrating fentanyl tablet in 12 patients (5.5\%). The most common adverse events were nausea, somnolence, dizziness, and vomiting. Twenty-one deaths occurred during the study period, but none were thought to be related to use of sublingual fentanyl.

\section{Open-label studies}

Rauck et al reported a multicenter, nonrandomized, openlabel Phase III study to evaluate the long-term effectiveness and tolerability of sublingual fentanyl for treatment of BTP. ${ }^{41}$ Patient eligibility was consistent with the previous Phase III trials which included patients with stable cancerrelated pain who were experiencing 1-4 breakthrough episodes a day. All patients were on fixed-schedule opioid regimens equivalent to 60-1000 $\mathrm{mg}$ /day of oral morphine or 50-300 $\mu \mathrm{g} /$ hour of transdermal fentanyl and had an Eastern Cooperative Oncology Group (ECOG) performance status of $0-2$.

Patients initially completed a 2 -week dose titration phase followed by a maintenance phase of up to 12 months. Patients were titrated from a dose of $100 \mu \mathrm{g}$ sublingual fentanyl to a maximum of $800 \mu \mathrm{g}$ until an effective dose was reached. This was defined as a dose that provided adequate pain relief without intolerable adverse events for two consecutive days. Once an effective dose was obtained, patients then entered the open-label maintenance phase. During this phase, patients used sublingual fentanyl for BTP episodes, with a minimum of 2 hours between study medications. If adequate relief was not obtained, rescue medication could be used within 2 hours of treatment. Efficacy was evaluated using quality of life and patient satisfaction data as measured by the Patient Global Evaluation of Medication, the Brief Pain Inventory, and the Depression, Anxiety, and Positive Outlook Scale.

In total, 139 patients entered the titration phase and 96 patients entered the long-term maintenance phase. Eighty-seven patients completed the Patient Global Evaluation of Medication at both screening and at the end of the study. The titration phase revealed a median effective dose of $400 \mu \mathrm{g}$ and a mean dose of $475 \mu \mathrm{g}$ in a range of $100-800 \mu \mathrm{g}$. The results of the Patient Global Evaluation of Medication at screening and at the end of the study, as well as at the 6-month visit, demonstrated a significant increase in reported satisfaction with use of the study medication in comparison with satisfaction on previous analgesic therapy at the time of study enrollment $(P=0.01)$. Mean Brief Pain Inventory scores for pain relief also demonstrated significant improvement at the 6-month and end of study visit $(P<0.05)$. Depression, Anxiety, and Positive Outlook Scale scores showed significant improvement in depression at 6 months $(P=0.011)$. Five of seven aspects of daily functioning recorded by the Brief Pain Inventory showed significant differences at 6 months, but only "life enjoyment" was significant at the end of the study visit $(P=0.02)$.

During both phases of this study, data on safety were collected. Of 139 patients who received at least one dose of sublingual fentanyl, $116(83.5 \%)$ reported at least one adverse event. The most common adverse events were nausea (23\%), 
fatigue (15.1\%), and vomiting (12.9\%). Adverse events were considered likely to be related to sublingual fentanyl in 49 of 139 patients (53.3\%). Serious adverse events occurred in 46 patients, but none of these were believed to be secondary to sublingual fentanyl use.

\section{Randomized, double-blind, controlled trials}

Rauck et al conducted a multicenter, double-blind, placebocontrolled trial to evaluate the efficacy and long-term tolerability of sublingual fentanyl tablets compared with placebo for BTP. ${ }^{42}$ Adult patients with stable cancer-related pain who were experiencing 1-4 episodes of BTP daily were eligible for this study. All patients were on fixed-schedule opioid regimens equivalent to $60-1000 \mathrm{mg} /$ day of oral morphine or $50-300 \mu \mathrm{g} / \mathrm{hour}$ of transdermal fentanyl. In addition, all patients had an ECOG performance status of 0-2.

Patients initially completed an open-label dose titration phase to identify a single dose of fentanyl that provided adequate pain relief over two consecutive days without unacceptable adverse events. Doses ranged from $100 \mu \mathrm{g}$ to $800 \mu \mathrm{g}$, with a median effective dose of $600 \mu \mathrm{g}$. Following dose titration, 66 eligible patients entered the 2 -week double-blind phase. Patients were given seven doses of sublingual fentanyl at their previously identified effective dosage and three doses of placebo to use randomly for 10 episodes of BTP, with at least one dose of fentanyl separating each placebo dose. In addition, each dose was separated by a minimum of 2 hours. The primary outcome was the sum of pain intensity difference (SPID) from baseline until 30 minutes after treatment. Pain intensity was measured on an 11 -point scale $(0=$ no pain, $10=$ pain as bad as imaginable) and assessed immediately prior to treatment and at time intervals of $10,15,30$, and 60 minutes after treatment. Secondary endpoints were pain relief as rated on a five-point scale $(0=$ no relief, $4=$ complete relief), SPID over 60 minutes, successful responders, use of rescue medication (which could be taken within 2 hours of the study drug intake if pain relief was inadequate), and overall patient satisfaction with the medication (using the Patient Global Evaluation of Medication five-point scale [1 = excellent, 5 = poor $]$ ).

In total, 131 patients entered the titration phase, 78 patients completed titration, 66 patients entered the double-blind efficacy phase, and 60 patients chose to enter the long-term safety phase. The primary outcome revealed a significantly greater difference in mean SPID at 30 minutes post-dose with use of sublingual fentanyl versus placebo $(P=0.0004)$. This was maintained at 60 minutes post-dose $(P=0.0002)$. Consistent with these results, sublingual fentanyl was found to provide greater pain relief than placebo starting at 10 minutes post-dose and maintained throughout the 60-minute evaluation $(P<0.049)$. The mean Patient Global Evaluation of Medication score was better with use of sublingual fentanyl than with the placebo (3.1 versus 3.6, $P=0.0006$ ), and almost half of the patients reported satisfaction with medication. The percentage of responders, defined as a reduction in pain intensity of at least $30 \%$ compared with baseline, was greater for sublingual fentanyl than placebo (86.9\% versus $64.9 \%$ ). Fewer episodes required rescue medication with use of sublingual fentanyl than use of placebo (11.2\% versus $27.4 \%)$

This study included an open-label safety phase for up to 12 months, during which time patients continued use of sublingual fentanyl for cancer-related BTP. Of the initial 131 patients, 96 patients experienced at least one adverse event. The most common drug reactions were nausea (12.2\%), vomiting (5.3\%), and somnolence (4.6\%). All patients who completed the double-blind phase of the study elected to continue into the safety phase of the trial. There were 24 serious adverse events and 10 deaths during the study, but only one serious adverse event, mild affect lability, was considered to be possibly secondary to sublingual fentanyl administration.

\section{Buccal fentanyl tablets}

Two randomized, placebo-controlled trials have been performed to evaluate the efficacy and tolerability of buccal fentanyl tablets for BTP. Portenoy et al conducted a double-blind, randomized, placebo-controlled trial to evaluate the efficacy and tolerability of buccal fentanyl tablets for the relief of cancer-related BTP in opioid-tolerant patients. ${ }^{43}$ Adult cancer patients currently on opioid therapy who were experiencing 1-4 episodes of BTP daily were eligible for this study. All patients were required to be currently on an opioid regimen equivalent to 60-1000 mg/day oral morphine or 50-300 $\mu \mathrm{g}$ /hour of transdermal fentanyl. In addition, all patients had to have an ECOG performance status rating between 0 and 2 and a life expectancy greater than 3 months.

Patients initially completed an open-label dose titration phase to identify a single dose of fentanyl that provided adequate pain relief within 30 minutes for two consecutive BTP episodes without unacceptable adverse events. Dosing was initiated at $100 \mu \mathrm{g}$, with titration up to a maximum dose of $800 \mu \mathrm{g}$. Once a satisfactory dose was reached, patients could then enter the double-blind phase. During this phase, patients were given seven doses of sublingual fentanyl at 
their previously identified effective dosage and three doses of placebo to use for 10 episodes of BTP in one of 18 predetermined sequences. If inadequate relief was obtained from the study medication within 30 minutes, patients were allowed to use supplemental medication. Each dose of study medication was separated by a minimum of 4 hours, and a maximum of four episodes was treated with study medication per day. The primary outcome was the SPID from baseline until 30 minutes after treatment. Pain intensity was measured on an 11 -point scale $(0=$ no pain, $10=$ worst imaginable pain $)$ and assessed immediately before treatment and at time intervals of 15,30,45, and 60 minutes after treatment. Secondary endpoints were pain intensity difference and pain relief, as rated on a five-point scale $(0=$ no relief, $4=$ complete relief) at each time point and as a sum of pain relief scores, global medication performance assessment ratings at 30 and 60 minutes using a five-point scale ( $0=$ poor, $4=$ excellent), use of supplemental medication after study medication, and number of treated episodes that demonstrated improvement of greater than $33 \%$ or greater than $50 \%$ in pain intensity scores at each time point.

In total, 123 patients entered the titration phase, 77 enrolled in the double-blind phase, and 68 completed the entire study. The median effective dose was $400 \mu \mathrm{g}$ and the mean dose was $475 \mu \mathrm{g}$, with a range of $100-800 \mu \mathrm{g}$. There was no apparent correlation between baseline opioid regimen or supplemental medication dose and effective buccal fentanyl tablet dose for each patient. The primary outcome in evaluating efficacy revealed that the SPID was significantly higher with use of buccal fentanyl tablets versus placebo at 30 minutes $(P<0.0001)$. In addition, the mean pain intensity difference, SPID, and pain relief scores were all significantly higher for buccal fentanyl tablet doses than for placebo at all time points $(P<0.003$ at 15 minutes, $P<0.0001$ at 30 , 45 , and 60 minutes). Global medication performance ratings were better with use of buccal fentanyl tablets than with placebo, ie, 1.4 versus 0.9 at 30 minutes and 2.1 versus 1.3 at 60 minutes $(P<0.0001)$. A higher percentage of patients had clinically significant improvement in pain scores $(>33 \%$ and $>50 \%$ ) when treated with buccal fentanyl tablets versus placebo. At 30 minutes, $48 \%$ of buccal fentanyl tablet-treated episodes showed a reduction of greater than $33 \%$ in pain scores, with $24 \%$ having a reduction of greater than $50 \%$ versus $29 \%$ and $16 \%$, respectively, for BTP episodes treated with placebo $(P<0.0001$ and $P=0.0023)$. Fewer episodes required use of supplemental medication when treated with buccal fentanyl tablets compared with use of placebo (23\% versus $50 \%)$.
Safety and tolerability of medication was assessed throughout both phases of this study. The most commonly reported adverse events were nausea (22\%), vomiting (11\%), dizziness (22\%), constipation ( $8 \%$ ), and somnolence (10\%). Adverse events led to withdrawal from the study in approximately $6 \%$ of patients. Two patients experienced mucosal ulcers at the site of administration of buccal tablets. There were 13 serious adverse events and seven deaths during this study, but none were considered related to use of the study medication.

For further evaluation of the efficacy and tolerability of buccal fentanyl tablets in BTP, Slatkin et al performed a similarly designed double-blind, randomized, placebo-controlled trial. ${ }^{44}$ Adult cancer patients currently on opioid therapy who were experiencing 1-4 episodes of BTP daily without recent escalation were eligible for this study. All patients were required to be currently on an opioid regimen $\geq 60 \mathrm{mg}$ /day of oral morphine or $25 \mu \mathrm{g} /$ hour of transdermal fentanyl. In addition, all patients had to have a life expectancy of at least 2 months.

Patients initially completed an open-label dose titration phase to identify a single dose of fentanyl that provided adequate pain relief within 30 minutes for two consecutive BTP episodes without unacceptable adverse events. Dosing was initiated between $100 \mu \mathrm{g}$ and $800 \mu \mathrm{g}$, based on the breakthrough medication used prior to enrollment in the study, then titrated up to the next higher dose until a satisfactory dose was reached. Once an adequate dose was found, patients then entered the double-blind phase. During this phase, patients were treated for 10 episodes of BTP in one of 18 predetermined sequences using seven doses of sublingual fentanyl at their previously identified effective dosage and three doses of placebo. If inadequate relief was obtained from the study medication within 30 minutes, patients were allowed to use supplemental medication. A minimum of 4 hours was required between each dose of study medication. The primary outcome was the SPID from baseline until 60 minutes after treatment. Pain intensity was measured on an 11 -point scale $(0=$ no pain, $10=$ worst pain $)$ and assessed immediately prior to treatment with the study medication and at time intervals of $5,10,15,30,45,60$, 90, and 120 minutes after treatment. Secondary endpoints were pain intensity difference and pain relief as rated on a five-point scale $(0=$ no relief, $4=$ complete relief $)$ at each time point and as a sum of pain relief scores, the number of episodes requiring use of supplemental medication, the number of treated episodes that demonstrated improvement in pain intensity of greater than $33 \%$ or greater than $50 \%$, and global medication performance assessment ratings at 
30 and 60 minutes post-treatment using a five-point scale ( 0 = poor, $4=$ excellent $)$.

Overall, 129 patients entered the dose-titration phase and 87 patients achieved a satisfactory dose of buccal fentanyl tablets and continued into the double-blind phase. The effective dose was $100 \mu \mathrm{g}$ in $8 \%, 200 \mu \mathrm{g}$ in $12 \%, 400 \mu \mathrm{g}$ in $18 \%$, $600 \mu \mathrm{g}$ in $28 \%$, and $800 \mu \mathrm{g}$ in $34 \%$. There was not a clear association between baseline opioid regimens and effective buccal fentanyl tablet dose for each patient. The primary outcome in evaluating efficacy revealed that the SPID was significantly higher with use of buccal fentanyl tablets versus placebo over 60 minutes $(P<0.0001)$. In addition, the reduction in pain intensity was significantly different for buccal fentanyl tablets versus placebo starting at 10 minutes ( 0.9 versus $0.5, P<0.0001)$, and this was maintained through the entire observation period of 120 minutes $(P<0.0001)$. Accordingly, pain relief scores were also significantly higher for buccal fentanyl tablet doses than placebo at all time points after 10 minutes $(P<0.0001)$. Episodes requiring use of supplemental medication when treated with buccal fentanyl tablets were less than with use of placebo (11\% versus $30 \%$ ). The global medication performance ratings were better with use of buccal fentanyl tablets than with placebo, ie, 2.1 versus 1.2 at 60 minutes and 2.3 versus 1.2 at 120 minutes $(P<0.0001)$. A higher percentage of patients had clinically significant improvement in pain scores $(>33 \%$ and $>50 \%)$ when treated with buccal fentanyl tablets versus placebo. At 30 minutes, $51 \%$ of buccal fentanyl tablet-treated episodes had a reduction of greater than $33 \%$ in pain scores, with $38 \%$ having a reduction of greater than $50 \%$ versus $26 \%$ and $15 \%$, respectively, for BTP episodes treated with placebo $(P<0.0001)$.

During this study, $66 \%$ of patients reported adverse events, but most were mild and similar to those observed with all opioids. The most commonly observed adverse events were nausea (13\%), dizziness (11\%), and fatigue (8\%). Approximately $10 \%$ of patients experienced adverse events related to site of application, but most were transient in nature, and only one patient withdrew from the study secondary to this adverse event. Eleven patients had serious adverse events, including nine deaths; however, all were related to disease progression and unrelated to use of study medication.

\section{Fentanyl transmucosal tablets as breakthrough analgesia: use with other opioids}

Mercadante et al reported the results of a prospective study evaluating the efficacy of buccal fentanyl tablets for BTP in patients using methadone for their analgesic regimen. ${ }^{45}$ Thirteen consecutive patients with advanced cancer admitted to a palliative care unit on stable doses of methadone were observed. Patients were eligible if they were experiencing three or fewer pain episodes daily.

Patients enrolled in this study were instructed to alert nursing staff when severe pain ( $\geq 7 / 10$ on a 10 -point scale) occurred. They were then given buccal fentanyl tablets, dosed according to their background dose of methadone. A patient on $12 \mathrm{mg}$ of methadone daily received $100 \mu \mathrm{g}$ of buccal fentanyl $t$, with proportionally higher doses of buccal fentanyl given for patients on higher doses of methadone. For each episode, trained nurses recorded pain intensity on a numeric scale of 0-10 immediately prior to the buccal fentanyl tablets and 15 minutes after administration. Effective treatment was defined as a decrease in pain intensity by at least 33\% at 15 minutes and not requiring any additional treatment for 2 hours following administration. If patients did not have successful relief of symptoms at 15 minutes, they were offered intravenous methadone for supplemental medication.

In the 13 enrolled patients, 64 episodes of BTP were treated using buccal fentanyl tablets. Of these episodes, 20 resulted in at least a $33 \%$ decrease in pain intensity $(31.5 \%)$, and an additional 26 episodes resulted in at least a $50 \%$ decrease in pain intensity at 15 minutes $(40.6 \%)$. There were 10 episodes for which data was missing and nine episodes that were unsuccessfully treated (14\%), requiring supplementation with intravenous methadone. There was not a discernible increase in adverse events.

\section{Other opioids: buccal morphine and transdermal fentanyl}

Weinstein et al conducted a multicenter, nonrandomized, open-label study to evaluate the long-term tolerability of buccal fentanyl tablets for treatment of breakthrough cancer pain. ${ }^{46}$ Adult patients with cancer-related pain who were either buccal fentanyl tablet-naive or who had participated in one of two randomized controlled trials of buccal fentanyl tablets were eligible for this study. All patients were experiencing 1-4 episodes of BTP daily. Patients were required to have fixed-schedule opioid regimens equivalent to $60-1000 \mathrm{mg} /$ day of oral morphine or $50-300 \mu \mathrm{g} / \mathrm{hour}$ of transdermal fentanyl and a life expectancy of at least 2 months.

Patients who were buccal fentanyl tablet-naive initially completed a 2-week dose titration phase. During titration, dosing was initiated at $100 \mu \mathrm{g}$ and increased until a successful 
dose was reached up to a maximum of $800 \mu \mathrm{g}$. A successful dose was defined as a single dose of fentanyl that provided adequate pain relief within 30 minutes for two consecutive BTP episodes without unacceptable adverse events. Once an effective dose was obtained, these patients, as well as patients who had participated in the previous studies, entered the open-label maintenance phase for up to 12 months. During this phase, patients self-administered buccal fentanyl tablets at their previously established doses for BTP episodes. If adequate relief was not obtained within 30 minutes, a second buccal fentanyl tablet could be used. If repeated doses were needed for two of three BTP episodes per day, the dose could be increased. Up to six BTP episodes could be treated with buccal fentanyl tablets per day, with a daily maximum of eight buccal fentanyl tablets. Safety was the primary endpoint and was monitored by physical and neurological examinations, examination of the oral mucosa, and clinical laboratory tests every 3 months. In addition, monthly vital signs and continuous recording of adverse events were obtained. Secondary outcomes included a seven-item Patient Assessment of Medication following one month of use, the global medication performance questionnaire, and evaluation of need for dose changes.

A total of 232 patients entered the study, with 110 buccal fentanyl tablet-naïve patients completing the initial titration phase. The maintenance phase included 77 newly titrated patients and 120 patients who had undergone previous buccal fentanyl tablet titration. Overall, $90 \%$ of patients experienced at least one side effect; however, a majority of these were mild and deemed unrelated to use of buccal fentanyl tablets. During the maintenance phase, $38 \%$ of patients had an adverse event related to buccal fentanyl tablets. The most common were nausea $(10 \%)$, constipation $(8 \%)$, dizziness $(6 \%)$, and somnolence $(6 \%)$. In addition, 15 patients $(6 \%)$ had at least one adverse application site event, including pain, irritation, ulcer, or paresthesia. Throughout the entire study, adverse events led to withdrawal of 77 patients (33\%), but this was secondary to disease progression in 53 cases. Only one serious adverse event, ie, drug withdrawal syndrome, was attributable to buccal fentanyl tablets. Sixty deaths occurred during the study, but none were related to use of buccal fentanyl tablets. There were no remarkable findings in regard to laboratory values or changes in physical examinations. Most patients were able to maintain their initial dosage of buccal fentanyl tablets throughout the study (136/197), with an increased dose required for 54 patients and a lower dose for seven patients. Patient assessment of the medication revealed that a majority of patients preferred buccal fentanyl tablets to their previous BTP medication with regard to overall preference ( $88 \%$ versus $12 \%$ ), time to onset of pain relief (95\% versus $5 \%$ ), ease of administration ( $66 \%$ versus $34 \%$ ), and convenience of use ( $68 \%$ versus $32 \%$ ). In addition, most patients rated buccal fentanyl tablets as good or very good according to the global medication performance survey, with stability in these ratings throughout the maintenance phase and at the end of the study.

\section{Method of administration}

Fentanyl tablets are placed between the cheek and gums above the molar tooth and held for 10 minutes to allow dissolution. Any remaining tablets can be massaged against the cheek for another 5 minutes. Patients may drink 30 minutes after administration.

\section{Dosing}

The product information ${ }^{47}$ for transmucosal fentanyl tablets recommends a starting dose of $100 \mu \mathrm{g}$ for opioid-tolerant patients not transitioning from oral transmucosal fentanyl citrate. Table 2 shows the recommended starting doses for patients receiving oral transmucosal fentanyl citrate. It is recommended that if the BTP episode is not relieved after 30 minutes, patients should take only one additional dose at the same strength. Patients should wait at least 4 hours before treating another episode of BTP. Patients should take a maximum of two doses for any breakthrough episode. Patients must wait 4 hours before treating another episode of BTP. Clinicians should provide one strength of tablet at a time.

\section{Titration of buccal fentanyl tablets}

The product information for fentanyl buccal tablets ${ }^{47}$ recommends close monitoring of the patient by the clinician until the patient reaches their individual breakthrough dose. Patients starting at $100 \mu \mathrm{g}$ and needing to titrate to a higher dose can be told to take two $100 \mu \mathrm{g}$ doses with the next breakthrough episode, and if this dose does not work, the patient can place two $100 \mu \mathrm{g}$ tablets on each side of the mouth.

Table 2 Buccal fentanyl tablet dosing in patients taking transmucosal fentanyl lollipops

\begin{tabular}{ll}
\hline $\begin{array}{l}\text { Transmucosal fentanyl } \\
\text { Iollipop }(\mu \mathrm{g})\end{array}$ & $\begin{array}{l}\text { Initial buccal fentanyl tablet } \\
\text { dose }(\mu \mathrm{g})\end{array}$ \\
\hline 200 & 100 \\
400 & 100 \\
600 & 200 \\
800 & 200 \\
1200 & $2 \times 200$ \\
1600 & $2 \times 200$ \\
\hline
\end{tabular}


Titration can continue using multiples of the $200 \mu \mathrm{g}$ tablets. Patients should not use more than four tablets simultaneously.

\section{Other dosing experiences}

The appropriate dosing of opioids, including buccal fentanyl tablets, for BTP remains controversial. In order to assess the efficacy and safety of using buccal fentanyl tablet doses based proportionally on background opioid doses for BTP in opioid-tolerant patients, Mercadante et al conducted a prospective study. ${ }^{48}$ Adult cancer patients with adequate pain control currently using opioid regimens equivalent to at least $500 \mathrm{mg}$ of oral morphine daily were recruited for this study.

Patients experiencing severe BTP ( $\geq 7 / 10$ on a 10 -point scale) received buccal fentanyl tablets proportional to their background opioid use. For example, a patient taking an equivalent of $600 \mathrm{mg}$ of oral morphine daily received $1000 \mu \mathrm{g}$ buccal fentanyl tablets for BTP episodes, whereas a patient taking an equivalent of $900 \mathrm{mg}$ of oral morphine daily received $1500 \mu \mathrm{g}$ buccal fentanyl tablets. For each episode, pain intensity, on a numeric scale of 0-10, was collected prior to and 15 minutes after buccal fentanyl tablet administration. The number of patients obtaining greater than $33 \%$ and greater than $50 \%$ reduction in pain intensity at 15 minutes was recorded. If patients had inadequate relief of symptoms, they were offered intravenous morphine as supplemental treatment. Unsuccessful treatment was defined as episodes that either did not achieve at least a $33 \%$ reduction in pain intensity at 15 minutes or required supplemental medication.

Twelve patients were recruited for this study, with a total of 79 episodes of BTP treated with buccal fentanyl tablets. Of these episodes, 14 resulted in at least a 33\% decrease in pain intensity and an additional 48 episodes resulted in at least a $50 \%$ decrease in pain intensity at 15 minutes. There were 11 episodes for which data were missing and six episodes that were unsuccessfully treated. The mean dose of buccal fentanyl tablets was $2233 \mu \mathrm{g}$, and the dose of buccal fentanyl tablets did not correlate with reduction of pain intensity. Dosing based on baseline opioid use was well tolerated, because there was not a marked increase or change in adverse events experienced, and all adverse events were mild.

\section{Conclusion}

The buccal fentanyl tablet appears to be an option for the management of breakthrough pain given its rapid onset of action, which fits the time characteristics of many types of breakthrough pain. Available and effective by multiple routes, it has greater bioavailability than the preceding fentanyl formulations. Further work is required with respect to comparison with other formulations, as well as optimal dosing methods for these formulations.

\section{Disclosure}

The authors report no conflicts of interest in this work.

\section{References}

1. Portenoy RK, Hagen NA. Breakthrough pain: definition, prevalence and characteristics. Pain. 1990;41(3):273-281.

2. Payne R. Recognition and diagnosis of breakthrough pain. Pain Med. 2007;8 Suppl 1:S3-S7.

3. Bruera E, Schoeller T, Wenk R, et al. A prospective multicenter assessment of the Edmonton staging system for cancer pain. J Pain Symptom Manage. 1995;10(5):348-355.

4. Bennett D, Burton AW, Fishman S, et al. Consensus panel recommendations for the assessment and management of breakthrough pain. Part 2: management. Pharm Ther. 2005;30(6):354-361.

5. Gomez-Batiste X, Madrid F, Moreno F, et al. Breakthrough cancer pain: prevalence and characteristics in patients in Catalonia, Spain. J Pain Symptom Manage. 2002;24(1):45-52.

6. Svendsen KB, Andersen S, Arnason S, et al. Breakthrough pain in malignant and non-malignant diseases: a review of prevalence, characteristics and mechanisms. Eur J Pain. 2005;9(2):195-206.

7. Mercadante S, Villari P, Ferrera P, Casuccio A. Optimization of opioid therapy for preventing incident pain associated with bone metastases. J Pain Symptom Manage. 2004;28(5):505-510.

8. Mantyh PW. Cancer pain and its impact on diagnosis, survival and quality of life. Nat Rev Neurosci. 2006;7(10):797-809.

9. Collins SL, Faura CC, Moore RA, McQuay HJ. Peak plasma concentrations after oral morphine: a systematic review. J Pain Symptom Manage. 1998;16(6):388-402.

10. Mercadante S, Villari P, Ferrera P, Bianchi M, Casuccio A. Safety and effectiveness of intravenous morphine for episodic (breakthrough) pain using a fixed ratio with the oral daily morphine dose. J Pain Symptom Manage. 2004;27(4):352-359.

11. Jackson K, Ashby M, Keech J. Pilot dose finding study of intranasal sufentanil for breakthrough and incident cancer-associated pain. J Pain Symptom Manage. 2002;23(6):450-452.

12. Coluzzi PH, Schwartzberg L, Conroy JD Jr, et al. Breakthrough cancer pain: a randomized trial comparing oral transmucosal fentanyl citrate (OTFC) and morphine sulfate immediate release (MSIR). Pain. 2001;91(1-2):123-130.

13. Zeppetella G. Sublingual fentanyl citrate for cancer-related breakthrough pain: a pilot study. Palliat Med. 2001;15(4):323-328.

14. Hagen NA, Fisher K, Stiles C. Sublingual methadone for the management of cancer-related breakthrough pain: a pilot study. J Palliat Med. 2007;10(2):331-337.

15. Fisher K, Stiles C, Hagen NA. Characterization of the early pharmacodynamic profile of oral methadone for cancer-related breakthrough pain: a pilot study. J Pain Symptom Manage. 2004;28(6):619-625.

16. Portenoy RK, Lesage P. Management of cancer pain. Lancet. 1999;353(9165):1695-1700.

17. Maguire P, Tsai N, Kamal J, Cometta-Morini C, Upton C, Loew G. Pharmacological profiles of fentanyl analogs at mu, delta and kappa opiate receptors. Eur J Pharmacol. 1992;213(2):219-225.

18. McClain DA, Hug CC. Intravenous fentanyl kinetics. Clin Pharmacol Ther. 1980;28(1):106-114.

19. Stanley TH, Hague B, Mock DL, et al. Oral transmucosal fentanyl citrate (lollipop) premedication in human volunteers. Anesth Analg. 1989;69(1):21-27. 
20. Zhang H, Zhang J, Streisand JB. Oral mucosal drug delivery: clinical pharmacokinetics and therapeutic applications. Clin Pharm. 2002;41(9):661-680.

21. Streisand JB, Varvel JR, Stanski DR, et al. Absorption and bioavailability of oral transmucosal fentanyl citrate. Anesthesiology. 1991;75(2):223-229.

22. Shaiova L, Lapin J, Manco LS, et al. Tolerability and effects of two formulations of oral transmucosal fentanyl citrate (OTFC; ACTIQ) in patients with radiation-induced oral mucositis. Support Care Cancer. 2004;12(4):268-273.

23. Stanley TH, Hague B, Mock DL, et al. Oral transmucosal fentanyl citrate (lollipop) premedication in human volunteers. Anesth Analg. 1989;69(1):21-27.

24. Weinberg DS, Inturrisi CE, Reidenberg B, et al. Sublingual absorption of selected opioid analgesics. Clin Pharm Ther. 1988;44(3):335-342.

25. Bredenberg $\mathrm{S}$, Nystrom $\mathrm{C}$. In-vitro evaluation of bioadhesion in particulate systems and possible improvement using interactive mixtures. J Pharm Pharmacol. 2003;55(2):169-177.

26. Lennerñas B, Hedner T, Holmberg M, Bredenberg S, Nystrom C, Lennerñas H. Pharmacokinetics and tolerability of different doses of fentanyl following sublingual administration of a rapidly dissolving tablet to cancer patients: a new approach to treatment of incident pain. Br J Clin Pharmacol. 2005;59(2):249-253.

27. Bredenberg S, Duberg M, Lennerñas B, et al. In-vitro and in-vivo evaluation of a new sublingual tablet system for rapid oromucosal absorption using fentanyl citrate as the active substance. Eur J Pharm Sci. 2003;20(3):327-334.

28. Sundell-Bredenberg S, Nystrom C. The possibility of achieving an interactive mixture with high dose homogeneity containing an extremely low proportion of a micronised drug. Eur J Pharm Sci. 2001;12(3):285-295.

29. Nystrom C, Westerberg M. The use of ordered mixtures for improving the dissolution rate of low solubility compounds. J Pharm Pharmacol. 1986;38(3):161-165.

30. Westerberg M, Nystrom C. Physicochemical aspects of drug release XVII. The effect of drug surface area coverage to carrier materials on drug dissolution from ordered mixtures. Int J Pharm. 1993;90(1):1-17.

31. Darwish M, Kirby M, Robertson P, Tracewell W, Jiang JG. Pharmacokinetic properties of fentanyl effervescent buccal tablets: a Phase I, open-label, crossover study of single-dose 100, 200, 400, and 800 [mu] $\mathrm{g}$ in healthy adult volunteers. Clin Ther. 2006;28(5): 707-714.

32. Darwish M, Kirby M, Robertson P, Hellriegel E, Jiang JG. Single-dose and steady-state pharmacokinetics of fentanyl buccal tablet in healthy volunteers. J Clin Pharm. 2007;47(1):56-63.

33. Darwish M, Kirby M, Robertson P Jr, Hellriegel E, Jiang JG. Comparison of equivalent doses of fentanyl buccal tablets and arteriovenous differences in fentanyl pharmacokinetics. Clin Pharm. 2006;45(8):843-850.

34. Darwish M, Kirby M, Robertson P, Tracewell W, Jiang JG. Absolute and relative bioavailability of fentanyl buccal tablet and oral transmucosal fentanyl citrate. J Clin Pharm. 2007;47(3):343-350.
35. Darwish M, Tempero K, Kirby M, Thompson J. Relative bioavailability of the fentanyl effervescent buccal tablet (FEBT) $1080 \mathrm{pg}$ versus oral transmucosal fentanyl citrate $1600 \mathrm{pg}$ and dose proportionality of FEBT 270 to 1300 [mu] g: A single-dose, randomized, open-label, three-period study in healthy adult volunteers. Clin Ther. 2006;28(5): 715-724.

36. Lecybyl R, Hanna M. Fentanyl buccal tablet: faster rescue analgesia for breakthrough pain? Future Oncol. 2007;3(4):375-379.

37. Darwish M, Kirby M, Robertson P, Tracewell W, Jiang JG. Absorption of fentanyl from fentanyl buccal tablet in cancer patients with or without oral mucositis: a pilot study. Clin Drug Investig. 2007;27(9):605-611.

38. England R, Maddocks M, Manderson C, Zadora-Chrzastowska S, Wilcock A. How practical are transmucosal fentanyl products for breakthrough cancer pain? Novel use of placebo formulations to survey user opinion. BMJ Support Palliat Care. 2011;1(3):349-351.

39. Lennerñas B, Frank-Lissbrant I, Lennerñas H, Kañlkner KM, Derrick R, Howell J. Sublingual administration of fentanyl to cancer patients is an effective treatment for breakthrough pain: results from a randomized Phase II study. Palliat Med. 2010;24(3):286-293.

40. Uberall MA, Muller-Schwefe GHH. Sublingual fentanyl orally disintegrating tablet in daily practice: efficacy, safety and tolerability in patients with breakthrough cancer pain. Curr Med Res Opin. 2011;(7): 1385-1394.

41. Rauck RL, Tark M, Reyes E, et al. Efficacy and long-term tolerability of sublingual fentanyl orally disintegrating tablet in the treatment of breakthrough cancer pain. Curr Med Res Opin. 2009;25(12): 2877-2885.

42. Rauck RL, Tark M, Reyes E, et al. Efficacy and long-term tolerability of sublingual fentanyl orally disintegrating tablet in the treatment of breakthrough cancer pain. Curr Med Res Opin. 2009;25(12): 2877-2885.

43. Portenoy RK, Taylor D, Messina J, Tremmel L. A randomized, placebo-controlled study of fentanyl buccal tablet for breakthrough pain in opioid-treated patients with cancer. Clin J Pain. 2006;22(9): 805-811.

44. Slatkin NE, Xie F, Messina J, Segal TJ. Fentanyl buccal tablet for relief of breakthrough pain in opioid-tolerant patients with cancer-related chronic pain. J Support Oncol. 2007;5(7):327-334.

45. Mercadante S, Ferrera P, Arcuri E. The use of fentanyl buccal tablets as breakthrough medication in patients receiving chronic methadone therapy: an open label preliminary study. Support Care Cancer. 2011;19(3):435-438.

46. Weinstein SM, Messina J, Xie F. Fentanyl buccal tablet for the treatment of breakthrough pain in opioid-tolerant patients with chronic cancer pain. Cancer. 2009;115(11):2571-2579.

47. Drug Information Online. http://www.drugs.com/search.php?searchte $\mathrm{rm}=$ fentanyl+buccal+tablets accessed 30 May 2012.

48. Mercadante S, Ferrera P, Adile C, Casuccio A. Fentanyl buccal tablets for breakthrough pain in highly tolerant cancer patients: preliminary data on the proportionality between breakthrough pain dose and background dose. J Pain Symptom Manage. 2011;42(3):464-469.
Patient Preference and Adherence

\section{Publish your work in this journal}

Patient Preference and Adherence is an international, peer-reviewed, open access journal focusing on the growing importance of patient preference and adherence throughout the therapeutic continuum. Patient satisfaction, acceptability, quality of life, compliance, persistence and their role in developing new therapeutic modalities and compounds to

\section{Dovepress}

optimize clinical outcomes for existing disease states are major areas of interest. This journal has been accepted for indexing on PubMed Central. The manuscript management system is completely online and includes a very quick and fair peer-review system. Visit http://www.dovepress.com/ testimonials.php to read real quotes from published authors. 